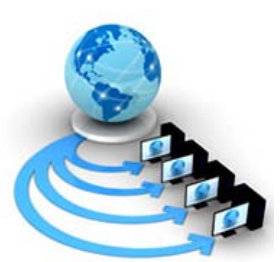

International Journal of Advanced Research in Computer Science

RESEARCH PAPER

Available Online at www.ijarcs.info

\title{
IMPROVED ACDCT AND PCA BASED DIGITAL IMAGE FUSION FOR VISULA SENSOR NETWORKS
}

\author{
Amandeep Singh \\ C.S.E\& G.N.D.U, \\ Amritsar,India
}

\author{
Sharanjeet Singh \\ Assistant Professor \\ C.S.E\& G.N.D.U Regional Campus, \\ Gurdaspur, ,India
}

\begin{abstract}
This paper has proposed another novel technique which has utilized the higher esteemed Alternating Current (AC) coefficients figured in Discrete Cosine Transform (DCT) domain based fusion with Principal Component Analysis(PCA) and adaptive histogram equalization out. The adaptive histogram equalization is utilized to improve the outcomes further and the utilization of PCA is for expanding the speed of the ACDCT based fusion strategy; in light of the fact that in color pictures AC-DCT has been connected on each color independently which is time devouring in nature. The trial comes about has unmistakably demonstrated that the proposed procedure beats over the accessible techniques. The proposed calculation has been planned and actualized in MATLAB apparatus utilizing picture preparing tool kit. The relative investigation has demonstrated the importance of the proposed calculation.
\end{abstract}

Keywords: Image Fusion, Discrete Cosine Transformation, Wavelet Transformation, Principle Component Analysis, Multi-focus images.

\section{INTRODUCTION}

In most sensor systems [1] - [8], every sensor can accomplish, make and trade data from anybody to another. Visual Sensor Networks recognizes a stage with two or three cameras that are acclimated to augment the assets and managing of a few focuses. In visual sensor arrange, sensors are cameras which can record either video grouping or pictures. The treatment of resultant information is connected to image processing. An outstanding component of visual sensor is to create vast measure of information. This component of visual sensor passes on simply the strong information that is symbolized at a conceptualized arrange. The goal of image fusion, is to diminish the aggregate sum of data amid arrange transmissions, is to create another picture that is more appropriate for additionally image processing errands.

Image fusion perform at three separate levels i.e. pixel, feature and decision, plans to accomplish the more right, total and dependable picture delineation of precisely the same. Pixel level is only a pitiable level of fusion which is used to audit and solidify data from particular sources previously special information is perceived. Feature level is only an inside level of fusion that wipes out a significant component from a picture like shape, length, segments, edges and course. Decision level is only an extremely anomalous condition of fusion that motivation to relate genuine objective. Picture fusion strategies may be generally masterminded into two methods i.e. spatial domain fusion and transform domain fusion. Spatial domain strategies are Averaging, Brovey strategy and Principal Component Analysis (PCA) techniques. Spatial domain picture fusion strategies are unpredictable and extended which are difficult to be performed on steady applications. Spatial domain strategies make distortion in the consolidated picture and this crisis may be dictated by transform domain method. At the point when this happens once the intertwined picture is spared in Joint Photographic Expert Group format, the fusion approaches which are associated in discrete cosine transform will be extraordinarily able. In transform domain technique, right off the bat picture is move into recurrence space. Discrete wavelet transform is a fusion system which performs under transform domain strategy [5]-[9].

\section{RELATED WORK}

R. Amutha et al. (2014) [9] has examined that basic and effective multi-center picture fusion system obviously got ready for remote visual sensor structure arranged with asset obliged, perilous setting like front lines. The fusion of multicenter pictures is engaged around higher regarded Alternating Current coefficients figured in Discrete Cosine Transform space. Discrete cosine change overcomes the calculation and vitality control of low power contraptions and is investigated similarly as picture quality and calculation vitality. It affirm the huge productivity upgrade of the proposed framework in yield quality and vitality utilization, when stood out from other fusion systems in DCT area. Vivek Kumar gupta et al. (2013) [10] has examined that in remote detecting program the raising alternative of space persisted sensors offer motivation to various picture fusion calculations. Remote detecting picture fusion design at organizing the information exchange by data gained which disguise particular components of the electromagnetic range at assorted spatial, temporal and spectral assurance; we can secure multi-temporal, multidetermination and multi-frequency picture information for plan behind element extraction, demonstrating and order. The fused picture is much serviceable for human data. Intertwined picture is more incredible for programmed PC examination undertaking for example instance feature extraction, segmentation and object recognization. Om parkash et al. (2013) [11] has investigated that the objective of picture fusion is to bring fitting information out of a few pictures of a similar zone in to a lone picture which is altogether informatory and is more suited to human data. Spatial domain based operations make spatial bends in the intertwined picture. Spatial domain distortion may be 
absolutely administered by the utilization of wavelet change based picture fusion forms. Utilizing incomparable most prominent fusion control wavelet coefficients at special deteriorating levels are fused. Two profound attributes wavelet symmetry and linear phase of BWT have just been took inclinations (exploited) for picture fusion in light of the way that they'll guarantee edge information. Uncertainties been exhibited that the wavelet change strategy enhance fusion quality by diminishing misfortune in fundamental information usable in single pictures. Shutao Li et al. (2013) [12] has broken down that a brisk and effective picture fusion procedures is proposed to make a profoundly enlightening intertwined picture through joining various pictures. Picture fusion strategy is established on a two-scale disintegration of a picture into an establishment layer containing huge scale varieties in power, and a spot of intrigue layer getting little scale points of interest. A novel guided separating based weighted normal technique is proposed to make full utilization of spatial consistency for fusion of the beds base and purpose of intrigue layers. It's been shown that the proposed framework can obtain best in class execution for fusion of multispectral, multi-center, multimodal, and multi-presentation pictures. Mohammed Hossny et al. (2013) [13] has examined that picture fusion philosophy consolidate different pictures into a man illuminating picture. Picture fusion measurements have made from picture preparing change measurements. In Image fusion measurements: advancement the main issue is the development of target picture fusion execution measurements and their subjective and objective acknowledgment. It clear up how fusion execution metric make beginning with picture distinction estimation, its comprehension into picture fusion associations, it confine weighting segment and the acknowledgment operation. Desale, R.P. et al. (2013) [14] has inspected the distinctive picture fusion strategies, for example, PCA (Principal Component Analysis), DCT (Discrete Cosine Transform) and DWT (Discrete Wavelet Transform) based picture fusion techniques. Creators have prescribed the determination of DWT based fusion technique for high caliber and precision applications. In this paper two calculations centered on DWT are proposed for instance, Pixel averaging and maximum pixel replacement algorithm. The execution of above said DWT's have been differentiated and the PCA and DCT fusion procedures. The examination has been completed centered on seven parameters named as PSNR, MSE, Normalize absolute error, Maximum Difference, Average Difference, Normalized CrossCorrelation and structural content. The outcomes depicts that the execution of DWT based fusion systems is through and through better as stand out from a substitute schedules for picture fusion. Y. Asnath et al. (2013) [15] has proposed a major and capable DCT based picture fusion strategy. Creators have prescribed the appropriation of this framework on a yard that DCT based fusion beats the calculation and vitality point of confinement of low power contraption. In this fusion technique, the picture hinders with higher estimation of AC coefficients is assimilated to the fused picture. It is great degree brisk as it does exclude any mind boggling gliding point number-crunching operations like mean or change figuring. The proposed fusion methodology fundamentally lessens the computational intricacy without exchanging off picture quality and for energy consumption dismemberment; it uses the ATmega 128 processor of Mica 2 bit at $8 \mathrm{MHz}$ with an effective compel use of $22 \mathrm{~mW}$ as the imminent state. The test outcomes show that the gigantic viability change of the proposed strategy in yield quality and vitality utilization, when appeared differently in relation to existing fusion frameworks, in DCT space. Sruthy, S et al. (2013) [16] has clarified a picture fusion systems utilizing Dual Tree Complex Wavelet Transform. In this fusion framework, fusion is performed utilizing the covers to focus information from the decayed structure of DT-CWT. Fusion procedure incorporates the arrangement of an intertwined pyramid using the DT-CWT coefficients which are obtained from the decayed pyramids of the info pictures. Finally the resultant intertwined picture is gained by applying the inverse dual tree complex wavelet transform. This outcome shows an immense decreasing of distortion. Yee Leung .et al. (2013) [17] has recommended that developing the adaptive intensity-hue-saturation procedure, an improved AIHS framework is proposed for pan sharpening. Through the IAIHS framework, the method for measuring spatial focuses infused into every one band of the multi-spectral picture is appropriately managed by weighting matrix that will be portrayed on the introduce of the edges of the panchromatic and MS pictures and the degrees including the MS bands. A realistic exhibits that the IAIHS system can keep spectral quality while giving practically identical spatial quality with the AIHS and added substance wavelet luminance relative techniques. Aribi, W. et al. (2012) [18] clarified that the idea of the restorative picture may be evaluated by few picture fusion methods. The fusion of pictures enhances the information to be set up by consolidating the information from chose pictures and the determination of fusion method depends on upon the application. For instance, this paper tends to the MRI and PET pictures. Here, eight picture fusion techniques alluded as Laplacian, FSD, Gradient, RATIO, Morph, Contrast, DWT and SIDWT frameworks have discussed. The parameters considered for the appraisal of results are Mutual Information, Universal Image quality Index and mean SSIM. The procured result exhibits that the RATIO and complexity systems present the best comes to fruition. Hugo R. Albuquerque et al. (2012) [19] has talked about that picture fusion is a property almost related to picture quality. In few pictures it's farfetched to acquire a reasonable concentration in many areas in the meantime, so a decision to make utilization of picture fusion to take an interest together pictures with differing center into bound together with all the present best-engaged locales. Two picture fusion calculations in the recurrence area which can be engaged around focus: separate on DCT domain and spatial frequency. The calculations isolate the picture in settled size pieces to choose which picture should surely be constituted the last come to fruition. Changes are made to the two techniques to choose when to pick a whole piece or pixels freely. The last picture quality, evaluated using PSNR and RMSE, is greatly improved set close by the results of the patient techniques. Kiran parmar et al. (2012) [20] has examined that the outline is to help the picture content by intertwining pictures like PC tomography and magnetic resonance imaging pictures; magnetic resonance imaging gives amazing information on sensitive tissue while registered tomography gives better data with respect to generous tissue. Combining those two sorts of pictures make 
a convoluted picture which is more enlightening than various signs gave by an individual methodology. Picture fusion has transformed into a consistent operation utilized inside medicinal diagnostics and treatment. Quick Discrete Curve let Transform utilizing Wrapper calculation based picture fusion strategy, has been execute, analyze and stood out from Wavelet based Fusion Technique. Fusion of pictures assembles at assorted purposes, control and by unmistakable frameworks causes specialist to pull back the qualities that may not be ordinarily obvious in an individual picture by various modalities. Rahul K Kher et al. (2012) [21] has broken down that the course of action is overhaul the picture content by combining pictures like PC tomography and attractive reverberation imaging pictures, keeping in mind the end goal to supply more information to the specialist and clinical treatment arranging framework. The goal is show the applying of wavelet change to multimodality medicinal picture fusion. This work secure the choice of wavelet function, the utilization of wavelet construct fusion calculations in light of medicinal picture fusion of PC tomography and therapeutic reverberation imaging, execution of fusion direction and the fusion picture standard evaluation. The fusion execution is engaged around the premise mean square error and peak signal to noise ratio. Pallavi Bute et al. (2012) [22] has talked about that picture fusion is the procedure in which a few pictures are intertwined to create new picture where every one of the things are center, to guarantee that circuit picture is more proper for human and machine perception. For therapeutic investigation reason the edges and graphs of the intrigued article are more basic than other information. To the extent this we proposed another therapeutic picture fusion design centered round blending of most prominent pixel substitution and complexity based strategy in wavelet space and their effect by changing the determination of picture. Haozheng, R et al. (2011) [23] has dissected that multi center picture fusion system centered on M-band MultiWavelet Transformation. For the motivation behind why, creators at first considered the multi center picture fusion methodology centered on single wavelet took after by multi wavelet, multi-band multi-wavelet along close by airtmetic of disintegration and recreation. For choosing fusion number-crunching directors, paper thinks concerning the systems centered on pictures, windows and zones. Also, pictures have been pondered centered around unmistakable fusion standard and assorted wavelets in the parts of entropy, peak SNR, square root error and standard mistake. Tentatively, it's been assumed that multiband multi wavelet is a doable picture fusion framework and gives favored execution over various methods. Mohamed, M et al. (2011) [24] has examined another way of picture fusion using Field Programmable Gate Arrays (FPGA). FPGA is an equipment based layout approach. FPGA system incorporates the equipment execution of the DCT, DWT and PCNN (beat coupled neural network) based fusion calculation. The trial result shows that FPGA based fusion technique give the better result as difference than existing picture fusion methodologies.

\section{VARIANCE IN LITERATURE SURVEY}

The related work on picture fusion calculations has demonstrated that the majority of existing calculations are tedious in nature and furthermore corrupts the brightness of the fused pictures. The principle purpose for this is a large portion of analysts have disregarded one of the accompanying or both:

1. No suitable picture improvement system is utilized to conquer the poor brightness of the intertwined picture.

2. No transform domain strategy is utilized to accelerate the fusion calculations.

3. No technique is considered for complex foundation pictures so it ends up plainly hard to fused pictures with complex foundation

\section{METHODLOGY}

To achieve the goal, well ordered system is utilized as a part of this thesis. Consequent are the diverse strides which are utilized to finish this work. Following are the different strides used to fulfill the targets of the paper 


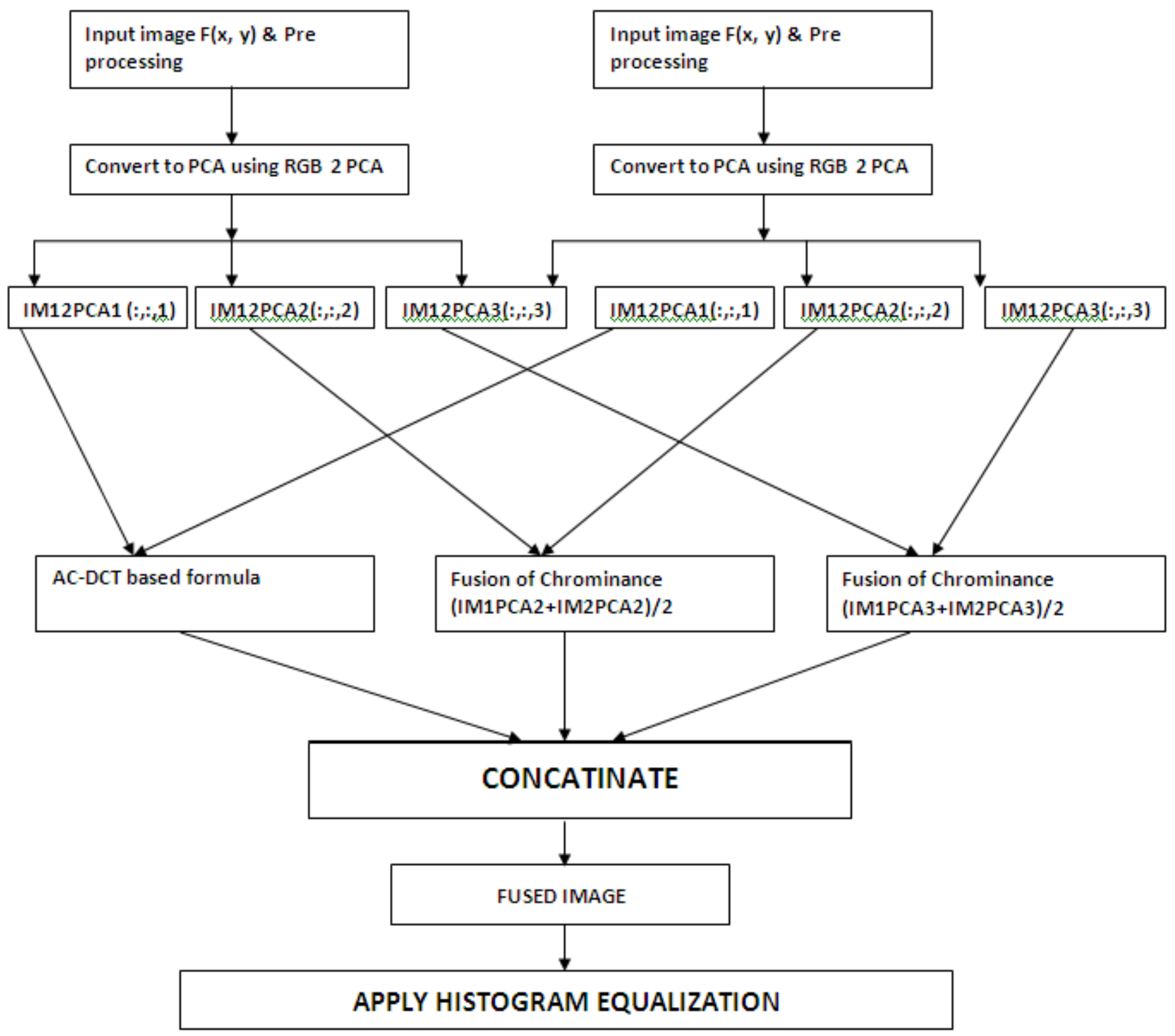

Fig. 1 Flowchart of proposed algorithm

The steps are as follows:

Step 1: Input images: Input 2 images image 1 and image 2 in which image $l$ is left blurred and image 2 is right blurred.

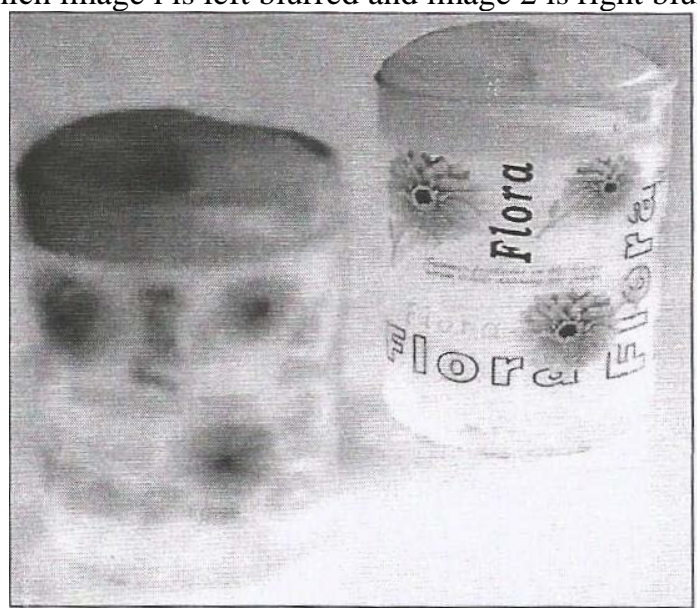

Fig 4 (a) Left blurred image

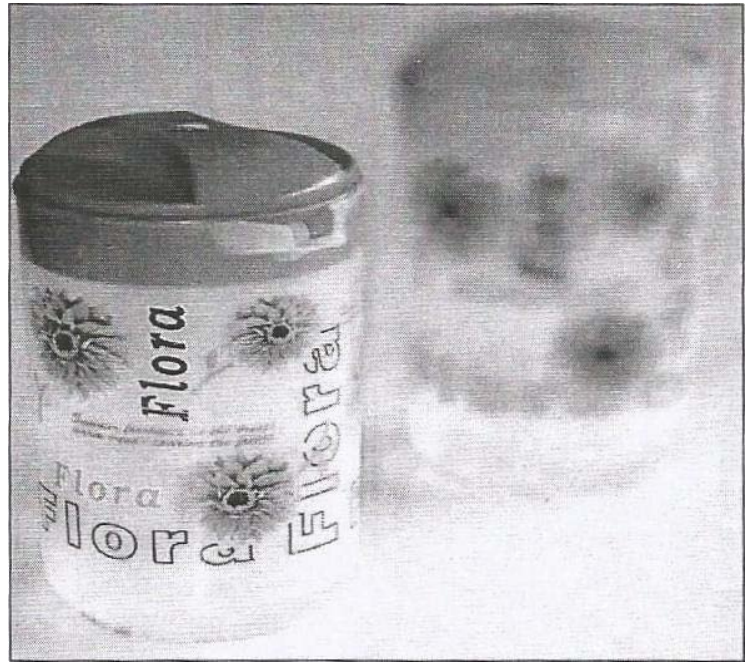

Fig 4(b) Right blurred image

Step 2: RGB2PCA: Presently RGB to PCA transformation will be done in view of the specific vector esteems. 
Likewise division of each PCA picture will likewise be done into PCA1, PCA2 and PCA3

Step3: Apply AC-DCT based fusion: The subsequent stage is to apply AC-DCT construct fusion with respect to first PCA as most elevated varieties found on the primary PCA plane. What's more, chrominance fusion will come in real life for other PCA planes i.e. PCA2 and PCA3.

Step4: Concatenation: Now concatenate the result of each plane and get the fused image.

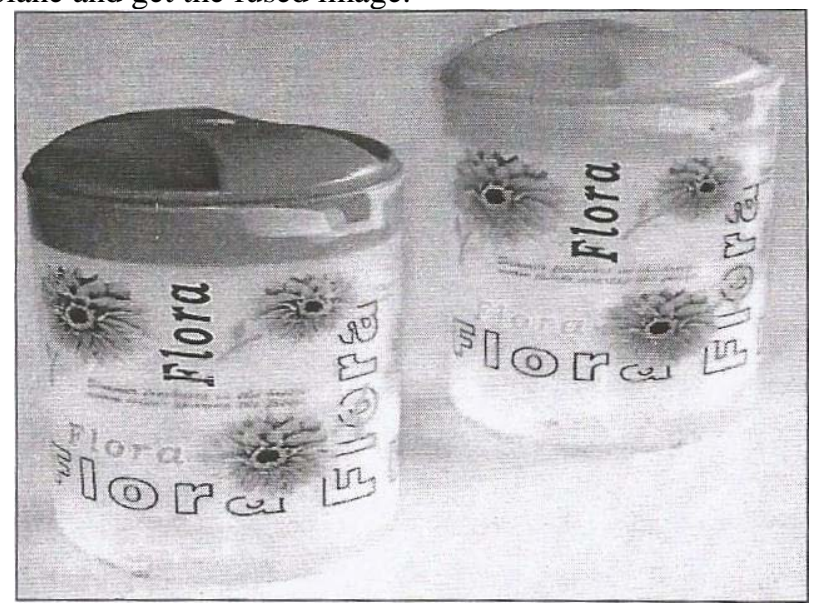

Fig 4: Final fused image

Step5: Adaptive histogram equalization: Now adaptive histogram equalization will come in action to preserve the brightness of the fused image. We convert the original image to cosine transform. Then inverse cosine transform is applied to that image. When inverse cosine transform is applied then noise is occurred in fused image then adaptive histogram is applied to remove noise and color artifacts which will introduced due to transform domain method i.e. DCT

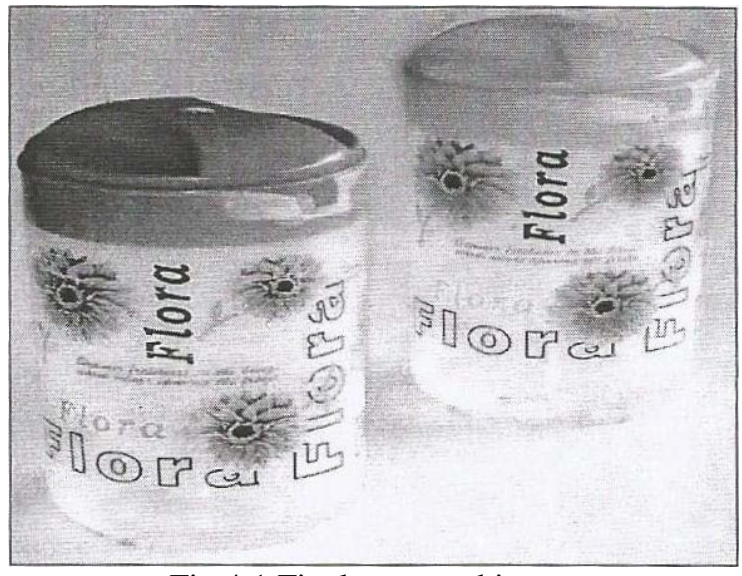

Fig 4:1 Final proposed image

\section{PERFORMANCE METRICS}

This area contains the cross approval really busy existing and proposed strategies. Some outstanding picture execution parameters for mechanized pictures have been shown that the execution of the proposed calculation is better than the present strategies. Some of parameters that are utilized are: Mean Square Error, Peak Signal Noise Ratio, Normalized Cross-Correlation, Average Difference and Root Mean Square Error

\section{Normalized Cross-Correlation (NCC)}

Normalized cross correlation is utilized to discover similarities between fused image and registered image is given by the following equation:

\section{Average Difference (AD)}

$$
\mathbf{N C C}=\sum_{i=1}^{m} \sum_{j=1}^{n}\left(\mathrm{~A}_{\mathrm{ij}}+\mathrm{B}_{\mathrm{ij}}\right)
$$

The Average maximum difference contrast relates to pixel which has a worth which is short of what the pixel in unique image and the Average minimum difference compares to pixel which has a quality which is more than the pixel in unique image. The average difference is characterized as an estimation of the distinction in the middle of maximum and minimum. It needs to be minimized.

$$
\mathrm{AD}=\frac{1}{m n} \sum_{i=1}^{m} \sum_{j=1}^{n}[\mathrm{~A}(\mathrm{i}, \mathrm{j})-\mathrm{B}(\mathrm{i}, \mathrm{j})]
$$

\section{Root mean square error (RMSE)}

Root mean square error between the remade image and the unique image presents mistake as a rate of the mean force of the first image. The RMSE is given by

$$
\sqrt{\frac{1}{M * N}} \sum x \sum y_{\left(\mathrm{I}_{\text {true }}(\mathrm{x}, \mathrm{y})-\mathrm{I}_{\text {fused }}(\mathrm{x}, \mathrm{y})\right)^{2}}
$$

Where $I_{\text {true }}(x, y)$ is the reference image, $I_{\text {fused }}(x, y)$ is the fusion image and $\mathrm{M}, \mathrm{N}$ are the dimensions of the images.

\section{EXPERIMENTAL RESULTS}

Fig.6.1 (a) is demonstrating the left blurred image and fig.6.1 (b) is demonstrating the right blurred image. The general target is to join significant data from various images into a solitary image that is more enlightening and suitable for both visual perception and further computer processing.

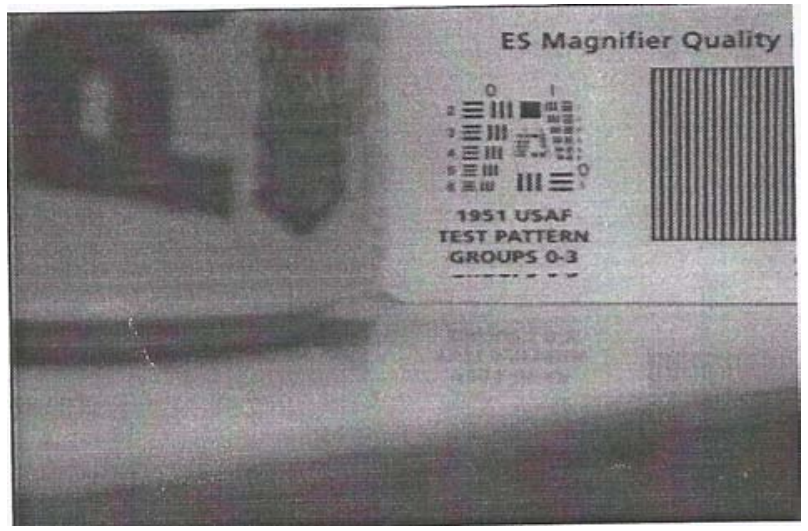

Fig. 6.1(a) Left blurred Image 


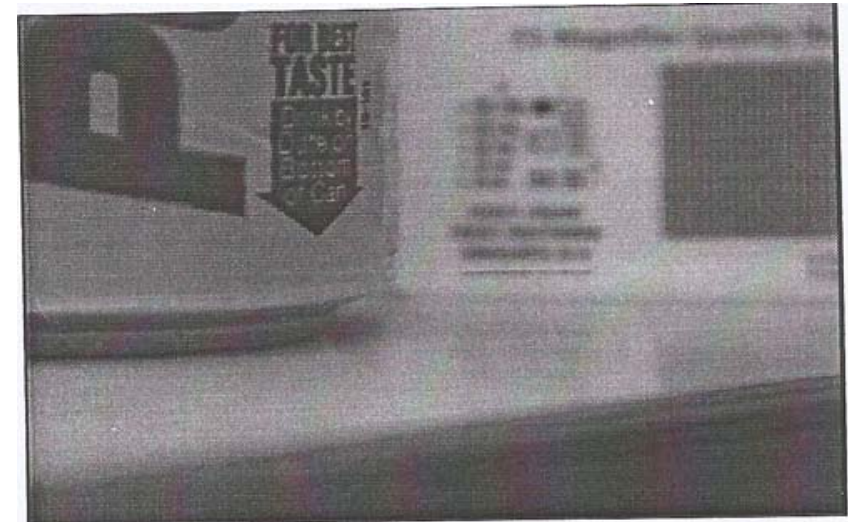

Fig. 6.1(b): Right blurred image

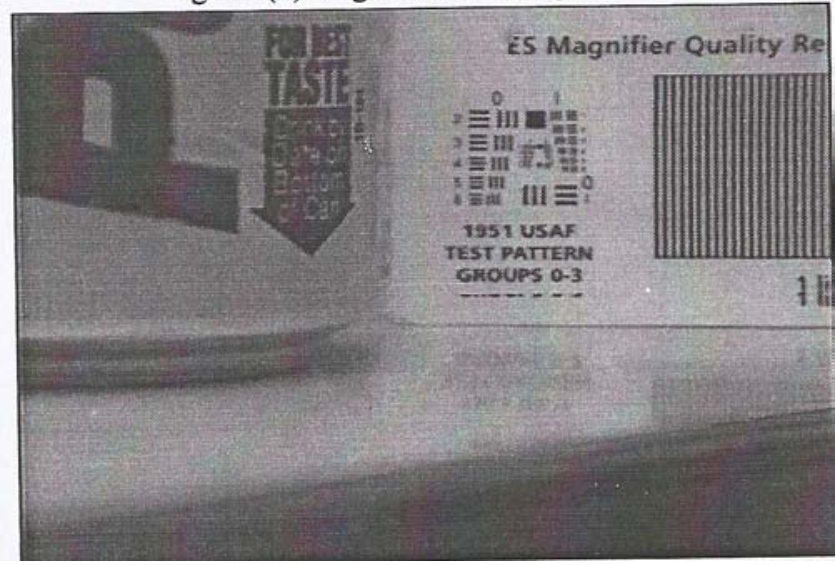

Fig. 6.2: Max-DCT based image fusion

Figure 6.2 has demonstrated the output image taken by Max-DCT. The output image has contained too much brightness and color unevenness as contrast with original blurred images to be fused. It is obviously demonstrated from the figure that the yield image is fused however some component has and also some arbitrary noise is likewise show in the images.

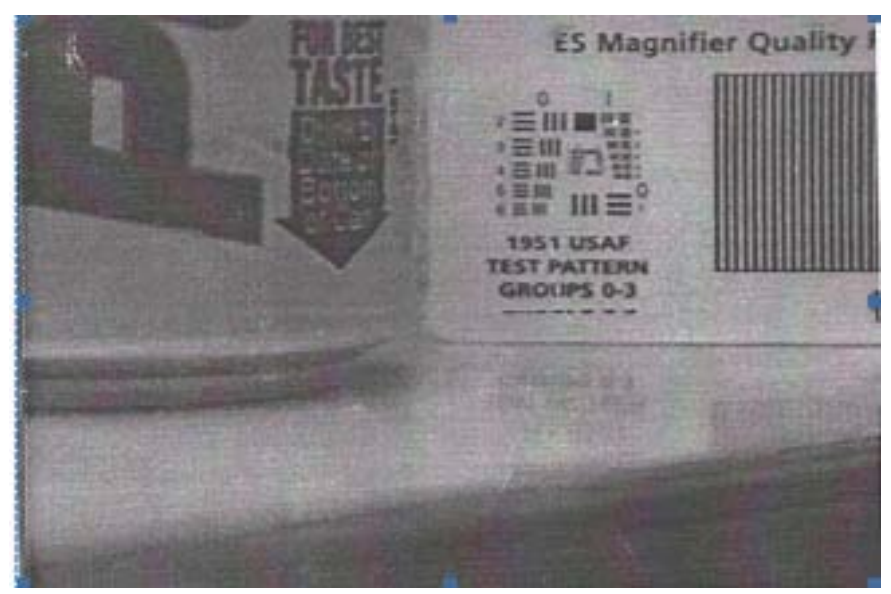

Fig.6.3: Final proposed image

Figure 6.3 has demonstrated the output image taken by the proposed image fusion with dynamic histogram stretching. The image has contained the adjusted color and brightness as the original images to be fused. The nature of output image is great with our proposed strategy regarding all the procedures examined.

\section{PERFORMANCE ANALYSIS OF PROPOSED SYSTEM}

This part contains the cross approval in the middle of existing and proposed methodology. Some outstanding picture execution parameters for modernized pictures have been shown that the execution of the proposed calculation is better than the present systems.

\subsection{Root Mean Square Error Evaluation}

Table 6.3 is demonstrating the quantized analysis of the root mean square error. As root mean square error needs to be lessened therefore the proposed algorithm is demonstrating the superior results than the accessible methods as mean square error is fewer in each case

Table.7.1 Root Mean Square Error Evaluation

\begin{tabular}{|l|l|l|l|}
\hline Images & Max-DCT & PCA & Proposed \\
\hline Image1 & 6.464162 & 43.370332 & 2.034873 \\
\hline Image2 & 8.890177 & 63.195079 & 2.973053 \\
\hline Image3 & 24.307532 & 85.355217 & 1.806115 \\
\hline Image4 & 14.792248 & 91.064418 & 6.893750 \\
\hline Image5 & 16.919651 & 103.059594 & 2.457786 \\
\hline Image6 & 25.638576 & 102.970553 & 14.084634 \\
\hline Image7 & 91.460178 & 136.400378 & 29.58929 \\
\hline Image8 & 52.655901 & 97.455123 & 16.483985 \\
\hline Image9 & 30.063252 & 82.258703 & 12.699037 \\
\hline Image10 & 54.384538 & 113.168143 & 11.70497 \\
\hline
\end{tabular}

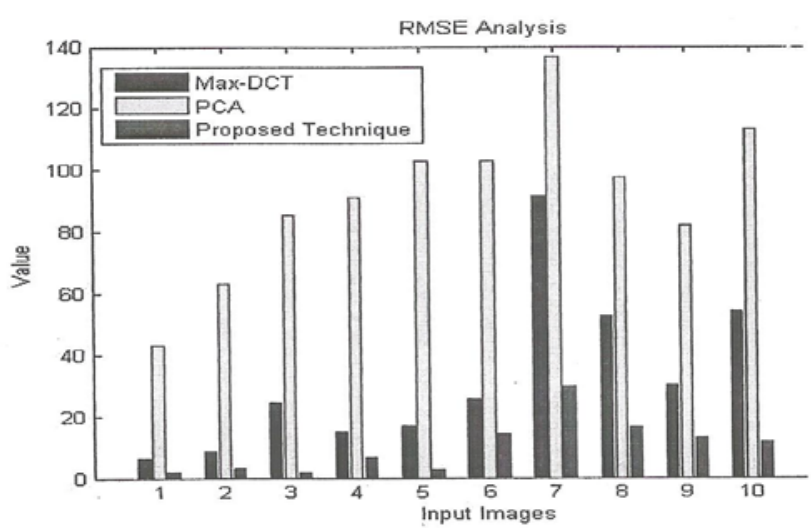

Fig.7.1: RMSE of MAX-DCT, PCA \& Proposed

Approach for different images

Figure 7.1 has demonstrated the quantized analysis of the root mean square error of distinctive images using fusion. It is clear from the plot that there is lessening in RMSE value of images with the use of proposed technique over different techniques. This diminishing symbolizes improvement in the goal nature of the image.

\subsection{Edge Preserving Index Evaluation}

Table 7.2 is demonstrating the similar analysis of the Edge Preserving Index (EPI). As EPI need to be expanded; so the fundamental objective is to expand the EPI however much as could be expected. Table 7.2 has clearly demonstrated that the EPI is most extreme on account of the proposed algorithm in this manner proposed algorithm is giving superior outcome than the available techniques. 
Table 7.2: Edge Preserving Index Evaluation

\begin{tabular}{|l|l|l|l|}
\hline Images & $\begin{array}{l}\text { Max- } \\
\text { DCT }\end{array}$ & PCA & Proposed \\
\hline Image1 & 0.7827 & 0.6856 & 0.9074 \\
\hline Image2 & 0.8539 & 0.6505 & 0.9201 \\
\hline Image3 & 0.9043 & 0.5826 & 0.9426 \\
\hline Image4 & 0.9472 & 0.6288 & 0.9786 \\
\hline Image5 & 0.9536 & 0.6422 & 0.9876 \\
\hline Image6 & 0.9343 & 0.2945 & 0.9831 \\
\hline Image7 & 0.7028 & 0.3039 & 0.9714 \\
\hline Image8 & 0.8429 & 0.5136 & 0.9144 \\
\hline Image9 & 0.9013 & 0.6516 & 0.9631 \\
\hline Imagel0 & 0.8757 & 0.4523 & 0.9663 \\
\hline
\end{tabular}

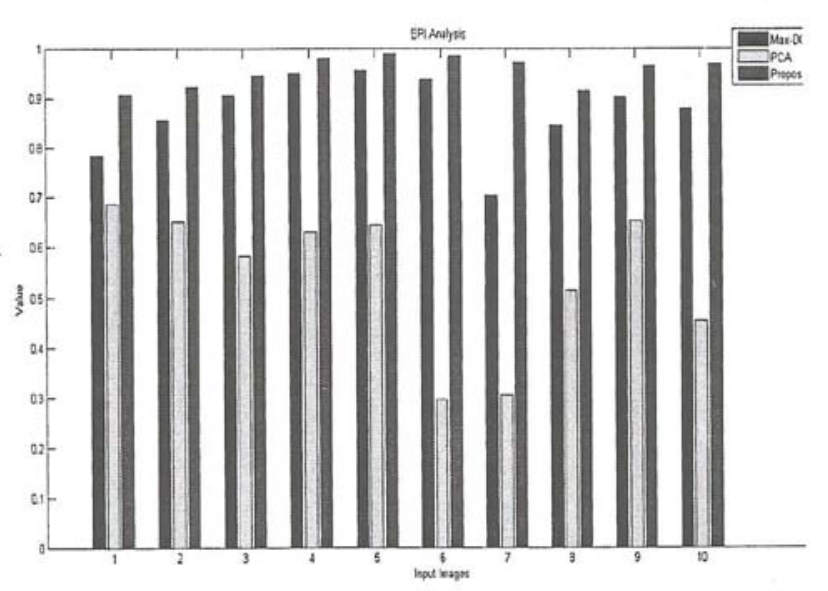

Fig. 7.2: EPI of MAX-DCT, PCA \& Proposed Approach for different images

Figure 7.2 has demonstrated the quantized analysis of the Edge Preserving Index of distinctive images using fusion. It is very clear from the plot that there is expand in EPI estimation of images with the use of proposed techniques over different strategies

\section{CONCLUSION AND FUTURE WORK}

The combination procedure may corrupt the sharpness of the intertwined pictures so to conquer this issue adaptive histogram equalization will be utilized to improve the outcomes further. The utilization of PCA is for expanding the speed of the AC-DCT based fusion strategy; in light of the fact that in color pictures AC-DCT has been connected on each color independently which is time expending in nature. To do the execution investigation distinctive measurements are considered in this thesis. The execution of picture combination is generally assessed as far as accuracy, PSNR and speed and so forth. The proposed system is composed and actualized in the MATLAB instrument utilizing picture preparing tool compartment. The test comes about has plainly demonstrated that the proposed procedure beats over the accessible strategies.

Image fusion observed to be the complex calculations as for the time complexity so in not so distant future we will propose another system that will utilize parallel processing to enhance the speed of the proposed calculation. Likewise the picture combination can likewise be additionally enhanced by utilizing the fuzzy set hypothesis to locate the best an incentive for intertwined picture from the information pictures to improve the outcomes further.

\section{REFRENCES}

[1] Albuquerque, Hugo R., Tsang Ing Ren, and George DC Cavalcanti. "Image Fusion Combining Frequency Domain Techniques Based on Focus." Tools with Artificial Intelligence (ICTAI), 2012 IEEE 24th International Conference on. Vol. 1. IEEE, 2012.

[2] Drajic, Dejan, and NedeljkoCvejic "Adaptive fusion of mu ltimodal surveillance image sequences in visual sensor networks." Consumer Electronics, IEEE Transactions on 53.4 (2007): 1456-1462.

[3] Desale, Rajenda Pandit, and Sarita V. Verma. "Study and analysis of PCA, DCT \& DWT based image fusion techniques" IEEE International Conference on Signal Processing Image Processing \& Pattern Recognition (ICSIPR), Coimbatore, pp. 66-69, 7-8 Feb., 2013.

[4] Tao, Ling, and Zhi-Yu Qian. "An improved medical image fusion algorithm based on wavelet transforms." Natural Computation (ICNC), 2011 Seventh International Conference on. Vol. 1. IEEE, 2011

[5] Zhang, Zhong, and Rick S. Blum. "A categorization of multiscal e-decompositionbased image fusion schemes with a performance study for a digital camera application." Proceedings of the IEEE 87.8 (1999): 1315-1326.

[6] Yang et al. EURASIP Journal on Advances in Signal Processing 2010 2010:579341

[7] Vladimir S. Petrovic , "Multisensor Pixel-level image Fusion," feb

[8] "DIP", [Lat visited] 27-august-2014, [ONLINEAVAILABLE]

http://www.edn.com/design/sensors/4397908/BiometricAuthentication--Multicue-data-fusionPart-I

[9] Phamila, Y., and R. Amutha "Discrete Cosine Transform based fusion of multi-focus images for visual sensor networks." Signal Processing 95 (2014): 161-170.

[10] Gupta, Vivek Kumar, AmitNeog, and S. K. Katiyar "Analysis of image fusion techniques over multispectral and microwave SAR images." Communications and Signal Processing (ICCSP), 2013International Conference on.IEEE, 2013.

[11] Prakash,Om, Richa Srivastava, and AshishKhare "Biorthogonal wavelet transform based image fusion using absolute maximum fusion rule." Information \& Communication Technologies (ICT), 2013 IEEE Conference on.IEEE, 2013.

[12] Li, Shutao, Xudong Kang, and Jianwen Hu. "Image fusion with guided filtering."IEEE transactions on image processing: a publication of the IEEE Signal Processing Society 22.7 (2013): 2864-2875.

[13] Hossny, Mohammed, et al "Image fusion metrics: evolution in a nutshell."ComputerModelling and Simulation (UKSim), 2013 UKSim 15th International Conference on.IEEE, 2013.

[14] Desale, Rajenda Pandit, and Sarita V. Verma. "Study and analysis of PCA, DCT \& DWT based image fusion techniques." In Signal Processing Image Processing \& Pattern Recognition (ICSIPR), 2013 International Conference on, pp. 66-69. IEEE, 2013.

[15] Y.AsnathVictyPhamila, R.Amutha. "Discrete Cosine Transform based fusion of multi-focus images for visual sensor networks." In Signal Processing, 2013 International Conference on, pp.161-170. IEEE, 2013.

[16] Sruthy, S., Latha Parameswaran, and Ajeesh P. Sasi. "Image Fusion Technique using DT-CWT." in Automation, Computing,

Communication, Control and Compressed Sensing (iMac4s), 2013 International Conference on, pp. 160-164. IEEE, 2013. 
[17] Leung, Yee, Junmin Liu, and Jiangshe Zhang. "An Improved Adaptive Intensity-Hue-Saturation Method for the Fusion of Remote Sensing Images." (2014): 1-5.

[18] Aribi, Walid, Ali Khalfallah, Med Salami Bouhlel, and Noomene Elkadri. "Evaluation of image fusion techniques in nuclear medicine." In Sciences of Electronics, Technologies of Information and Telecommunications (SETIT), 2012 6th International Conference on, pp. 875880. IEEE, 2012.

[19] Albuquerque, Hugo R., Tsang Ing Ren, and George DC Cavalcanti. "Image Fusion Combining Frequency Domain Techniques Based on Focus." Tools with Artificial Intelligence (ICTAI), 2012 IEEE 24th International Conference on. Vol. 1. IEEE, 2012.

[20] Parmar, K., \&Kher, R (2012, May). A comparative analysis of multimodality medical image fusion methods. In Modelling Symposium (AMS), 2012 Sixth Asia (pp. 9397),IEEE
[21] Parmar,kiran,RahulK.Kher, and Falgun N. Thakkar, "Analysis of CT and MRI Image Fusion UsingWavelet Transform." Communication Systems and Network Technologies (CSNT), 2012International Conference on. IEEE.2012

[22] Bute ,P and Sagar R. "Performance Evaluation of Contrast Based Image Fusion Technique Over Medical Image." In India Conference (INDICON) 2012 Annual IEEE, 2012, pp. 1184-1188

[23] Haozheng Ren, Yihua Lan, and Yong Zhang. "Research of Multi-Focus Image Fusion based on M- band Multi-Wavelet Transformation." In Fourth International Workshop on Advanced Computational Intelligence, 2011 International Conference on, pp.395-398.IEEE,2011.

[24] Mohamed ,M.A.,and B.M. EL-Den "Implementation of image fusion technique for multifocus images using FPGA." In radio science conference (NRSC) $201128^{\mathrm{Th}}$ national, pp.1-11. IEEE, 2011 